\title{
Acknowledgement to Reviewers of Metals in 2019
}

\author{
Metals Editorial Office \\ MDPI, St. Alban-Anlage 66, 4052 Basel, Switzerland \\ Published: 19 January 2020
}

The editorial team greatly appreciates the reviewers who have dedicated their considerable time and expertise to the journal's rigorous editorial process over the past 12 months, regardless of whether the papers are finally published or not. In 2019, a total of 1366 papers were published in the journal, with a median time to first decision of 13 days and a median time from submission to publication of 32 days. Among the reviewers who reviewed for Metals in 2019, the following five have been selected to receive the "Metals Outstanding Reviewer Award 2019" for the timeliness and quality of their review reports in 2019:

Dr. Antonio Riveiro from University of Vigo, Spain

Prof. Francisco J. G. Silva from Polytechnic Institute of Porto, Portugal

Dr. Javier Aldazabal from University of Navarra, Spain

Prof. Jose Diaz from University of Oviedo, Spain

Dr. Maciej Motyka from Rzeszow University of Technology, Poland

The editors also express their sincere gratitude to all of the following, who shared their time and expert opinion by reviewing for Metals in 2019:

\author{
Abbaschian, Reza \\ Abbott, Trevor \\ Abdollahnejad, Zahra \\ Abdolvand, Hamid \\ Abdul-Aziz, Ali \\ Abedini, Armin \\ Abella, Pura Alfonso \\ Abenojar, Juana \\ Abolfazl, Zahedi \\ Abramovich, Haim \\ Abrosimova, G. \\ Acar, Pınar \\ Acha, Victor \\ Actis Grande, Marco \\ Adachi, Shinichiro \\ Adachi, Yoshitaka \\ Adamek, Grzegorz \\ Adamiak, Marcin \\ Adamiec, Janusz \\ Adrian, Henryk \\ Affatato, Saverio \\ Afify, Ahmed Sabry \\ Aghion, Eli \\ Agostini, Matteo \\ Aguado, Sergio
}

\author{
Ahamed Khan, Riaz Ahamed \\ Ahmad, Hafiz Waqar \\ Ahmmed, K.M. Tanvir \\ Ahn, Byungmin \\ Ahuir-Torres, Juan \\ Akanda, Sajedur R. \\ Akhtar, Farid \\ Akiyama, Eiji \\ Akopyan, Torgom K. \\ Ala, Guido \\ Alaluss, Khaled \\ Alar, Vesna \\ Alavi, S. Habib \\ Albajez, Jose Antonio \\ Albizuri, Joseba \\ Aldazabal, Javier \\ Aldemir-Bektas, Basak \\ Aldwell, Barry \\ Alexandrov, Sergei \\ Alfano, Marco \\ Alfian, Ganjar \\ Ali, Usman \\ Alipour, Yousef \\ Allain, Sébastien Yves Pierre \\ Allen, J.B.
}


AlMangour, Bandar

Al-Saadi, Saad

Álvarez, Jose Alberto

Álvarez, José Alberto

Álvarez-Alonso, Pablo

Álvarez-Antolín, Jose Florentino

Alves de Sousa, Ricardo J.

Amado, Jose Manuel

Amanov, Auezhan

Ameth, Fall

Andersson, Anton

Andersson, Joel

Andersson, Nils

Ando, Yasutaka

Andreev, Dmitrii E.

Andriollo, Tito

Angella, Giuliano

Anggraini, Sri Ayu

Angulo, Carlos

Angurel, L.A.

Annett, Gebert

António Correia, José

Antoszewski, Bogdan

Antoun, Bonnie R.

Antretter, Thomas

Antunes, F. V.

Aoyagi, Kenta

Apopei, Andrei Ionut

Aranas, Clodualdo

Ardelean, Lavinia Cosmina

Arevalo, Cristina

Argatov, Ivan

Argilaga, Albert

Arias, Angel

Arias-Pardilla, Joaquín

Arrais, Aldo

Arrigoni, Michel

Arroyo Martínez, Borja

Artemev, Andrei

Artola, Garikoitz

Ashkenazi, Dana

Astarita, Antonello

Athanasiou, Christos

Attar, Hooyar

Atwater, Mark

Aubry, Pascal

Auger, Maria A.

Aune, Vegard

Aureliano, Manuel

Autrey, Tom

Avalle, Massimiliano

Avci, Recep
Aversa, Alberta

Awiszus, Birgit

Ayaso, Francisco-Javier

Azar, Amin S.

Bacciocchi, Michele

Bachagha, Tarek

Bache, Martin

Bachmaier, Andrea

Bachmann, Marcel

Badiola, Denis Jorge

Baffari, Dario

Bagherpour, Ebad

Bakaev, Alexander V.

Baker, Ian

Bała, Piotr

Balachandran Nair, Shanoob

Baláž, Matej

Balbo, Andrea

Ban, Masahito

Banakh, Oksana

Bani, Daniele

Bañón, Fermín

Bao, Jie

Baptista, Andresa

Barbaro, Frank

Barbieri, Giuseppe

Barbosa, Joaquim

Barella, Silvia

Barna, Martin

Barretta, Raffaele

Barriobero-Vila, Pere

Bartkowiak, Tomasz

Bartocha, Dariusz

Bartolome, Jose

Bartuli, Cecilia

Baruth, Andrew

Barylski, Adrian

Basaran, Cemal

Bassini, Emilio

Bäßler, Ralph

Batista, Moises

Baumeister, Joachim

Bay, François

Bayer, Ilker

Bayraktar, Emin

Bazlov, Andrey I.

Bazzicalupi, Carla

Beal, Coline

Beck, Tilmann

Beckermann, Christoph

Bedo, Tibor

Bedolla-Gil, Yaneth 


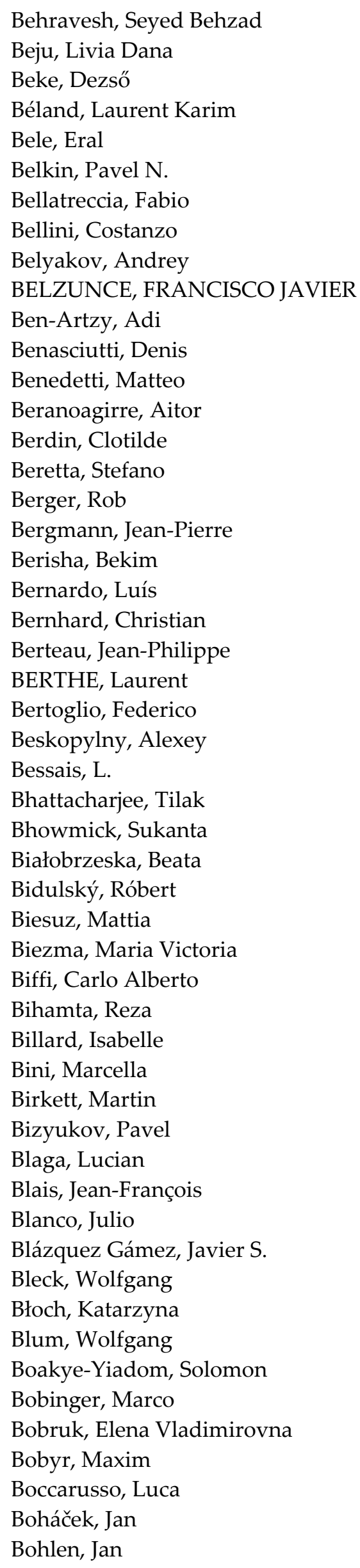

Böhm, Michał

Böhm, Stefan

Böhme, Marcus

Bolelli, Giovanni

Bolz, Sebastian

Bombac, David

Bonek, Mirosław S.

Bonse, Joern

Borchers, C.

Borchers, Christine

Bordeenithikasem, Punnathat

Borek, Wojciech

Borgioli, Francesca

Borla, Oscar

Bormashenko, Edward

Borodachenkova, Marina

Borodianskiy, Konstantin

Boshkov, Nikolai

Bossio, Antonio

Botto, Daniele

Boumerzoug, Zakaria

Brabazon, Dermot

Brahimi, Salim

Brailovski, Vladimir

Brajlih, Tomaž

Branco, Ricardo

Brandão, José A.

Brantley, William A.

Breaz, Radu Eugen

Brechtl, Jamieson

Breidenstein, Bernd

Breitbarth, Eric

Breslin, Carmel B

Brezinová, Janette

Brink, Tobias

Brnić, Josip

Brokmeier, Heinz-Günter

Brooks, Geoffrey

Bruder, Enrico

Bruni, Carlo

Brytan, Zbigniew

Bucchi, Francesco

Buchely, Mario

Bucinskas, Vytautas

Bugaev, Aram

Buhl, Johannes

Bui, Tinh Quoc

Buj Corral, Irene

Bulgariu, Laura

Bul'ko, Branislav

Bunaziv, Ivan

Bunn, Jeff 
Buonocore, Francesco

Burja, Jaka

Burkel, Eberhard

Burov, Sergey V.

Burpo, Fred J.

Bursik, Jiri

BUTT, JAVAID

Buzuk, Marijo

Bystrov, Vladimir

Cabeza Simo, Marta

Cabibbo, Marcello

Cabrera-Marrero, José-Maria

Cabrini, Marina

Cacko, Robert

Cahill, James

Cai, Feng

Calabrese, Luigi

Calero, Olga Caballero

Calvo Guirado, José Luis

Calvo-Guirado, José Luis

Camacho, Ana M.

Cambronero, Luis Enrique G.

Camelia, Gabor

Caminero Torija, Miguel Ángel

Campagnolo, Alberto

Campatelli, Gianni

Campbell, Archie

Campbell, John

Campi, Gaetano

Cannone Falchetto, Augusto

Capdevila-Montes, Carlos

Capela, Carlos Alexandre Bento

Caputi, Lorenzo S.

Carabineiro, Sonia

Cárcel-Carrrasco, Francisco Javier

Carlberg, Torbjorn

Carlone, Pierpaolo

Carou, Diego

Carpenter, Kristin

Carrado, Adele

Carron, Denis

Casagrande, Angelo

Casali, Dick

Casalino, Giuseppe

Casari, Daniele

Casati, Riccardo

Caschera, Daniela

Case, Raymundo

Castro Cerda, Felipe M.

Cavalli, Matthew

Cayron, Cyril

Čech, Jaroslav
Celada-Casero, Carola

Celikin, Mert

Cempura, Grzegorz

Cerchier, Pietrogiovanni

Cereceda, David

Cerri, Emanuela

Chalal, Hocine

Chalioris, Constantin

Challapalli, Suryanarayana

Champion, Yannick

Chan, Chi Wai

Chandola, Nitin

Chang, Kunok

Chang, Wenlong

Chantana, Jakapan

Charles, Yann

Chateigner, Daniel

Chaudhary, Anna Lisa

Chaus, Alexander S.

Chavoshi, Saeed Zare

Chekhonin, Paul

Chelgani, Saeed

Chen, Chuantong

Chen, Shuo

Chen, Shuying

Cherukara, Mathew

Chesneau, Xavier

Chicos, Lucia-Antoneta

Chirita, Bogdan

Chmelko, V.

Chmielewski, Tomasz

Cho, Hyunah

Cho, Jae-Hyung

Cho, Jong-Rae

Cho, Jung-Wook

Choi, Chul-Jin

Choi, SeongHo

Chotěborský, Rostislav

Christiansen, Thomas Lundin

Chrysanthou, Andreas

$\mathrm{Chu}$, Seok Jae

Chung, Hyun

Churyumov, Alexander Yu

Ciccotti, Giovanni

Ciceri, Davide

Cicero, Sergio

Cieslar, Miroslav

Cintas, Jesus

Ciocoiu, Robert Catalin

Citarella, Roberto G.

Ciuffini, Andrea Francesco

Cizek, Jan 
Cizelj, Leon

Clausmeyer, Till

Clayton, John D.

Coduri, Mauro

Coelho, Luís

Coenen, Jan Willem

Colla, Valentina

Collini, Luca

Collins, Sunniva

Colombini, Elena

Comesaña, Rafael

Concli, Franco

Cong, Weilong

Constantin, Lucian

Cooke, Kavian

Cooper, Daniel

Corbin, Stephen

Ćorić, Danko

Corigliano, Pasqualino

Correa, Cinthia Antunes

Corrêa, Edmilson

Correia, José A.F.O.

Cosson, Benoit

COTE, GERARD

Couque, Hervé

Courouau, Jean-Louis

Coy, Emerson

Crane, Nathan

Cristea, Dan

Croccolo, Dario

Crupi, Vincenzo

Csaki, Ioana

Csetenyi, Laszlo J.

Cuesta, Ivan

Cuevas, Francisco

Cui, Minming

Culliton, David

Ćurković, Helena Otmačić

Cwudziński, Adam

Cyr, Edward D

Czekanski, Aleksander

Czeppe, Tomasz

Cziegler, Andreas

Czinege, Imre

Czujko, Tomasz

Da Fonseca, Gláucio Soares

Dadić, Zvonimir

Dadkhah, Mehran

Daehn, Glenn

Dahal, Bishnu

Dahal, Jiba

Dahle, Sebastian
Dalla Vedova, Matteo D. L.

Dallago, Michele

Daloz, Dominique

Damma, Devaiah

Dancette, Sylvain

Danninger, Herbert

Darvishi Kamachali, Reza

Das, Aniruddh

Das, Manohar

Davim, J. Paulo

De Finis, Rosa

De Hosson, Jeff

De La Fuente, Germán

de la Fuente, Germán F.

De Lacalle, Luis N. López

De Luca, Alessandro

De Santis, Michele

Dehghan, Amir

Dehghanghadikolaei, Amir

Dehghan-Manshadi, Ali

Del Val, Jesús

Delannay, Francis

Delbe, Karl

Dell' Agli, Gianfranco

Demers, Vincent

Derlatka, Anna

Deshpande, Anagh

Dey, Poulumi

Di Mauro, Alessandro

Di Schino, Andrea

Dialami, Narges

Díaz Fernández, Belén

Diaz, Jose

Diaz, Marcos

Diehl, Martin

Diella, Valeria

Dieringa, Hajo

Diószegi, Attila

Dixon, David G.

Dixon, David A.

Djukic, Milos

Dlouhý, Ivo

Dobeš, Ferdinand

Dobrotă, Dan

Dobrzycki, Arkadiusz

Doidge, Euan

Dolata, Anna

Dománková, Mária

Doñate, Cristina Martín

Donchev, Alexander

Dong, Yan

Donik, Crtomir 
Doquet, Véronique

Dorafshan, Sattar

Dorin, Thomas

Dorogoy, Avraham

Drawin, Stefan

Drescher, Philipp

Drezet, Jean-Marie

Drozdenko, Daria

Drzazga, Michał

Duarte, Isabel

Duarte, Liliana

Dubey, Shri

Duchesne, Marc

Duchosal, Arnaud

Ducobu, François

Dudina, Dina V.

Dudko, Valeriy

Duisit, Jerome

Dulal, Rajendra

Dulska, Agnieszka

Duplakova, Darina

DuQuesnay, David

Durand, Bastien

Durejko, Tomasz

Dutta, Abhishek

Dutta, Biswanath

Dyl, Tomasz

Dymáček, Petr

Dymek, Stanisław

Dyzia, Maciej

Dzioba, Ihor

Dziubek, Kamil

Eakins, Daniel

Eason, Paul D

Ebna Hai, Bhuiyan Shameem Mahmood

Echterhof, Thomas

Ecker, Werner

Eckert, Maroš

Eckert, Sven

Economou-Eliopoulos, Maria

Edagawa, Keiichi

EFREMENKO, VASILY

Efthymiadis, $P$.

Efthymiadis, Panos

Egberts, Philip

Egorkin, Vladimir

Eisele, Timothy C.

Eisenbart, Miriam

Eisenlohr, Philip

Eklund, Per

Eksteen, Jacobus

El-Awady, Jaafar A.
Eliseev, Alexander

Ellendt, Nils

Elmi, Chiara

Elsaid, Khaled

Enesca, Ioan Alexandru

Engelberg, Dirk

Entezari Zarandi, Ali

Enz, Josephin

Enzinger, Norbert

Epp, Jeremy

Erdakov, Ivan Nikolaevich

Erdely, Petra

Eremin, Artem

Erisov, Yaroslav

Erna, Delczeg

Ernst, Frank

Errandonea, Daniel

Ersson, Mikael

Esin, Vladimir A.

Eslami, Hossein

Esposito, Luca

Evin, Emil

Facchini, Francesco

Falkus, Jan

Fallah, Vahid

Fan, Yong

Fargnoli, Mario

Farrokhpay, Saeed

Faruqi, M. A.

Fatyeyeva, Kateryna

Faulkner, R. G.

Fazakas, Eva

Fazeli, Fateh

Feldshtein, Eugene E.

Felgueiras, Helena P.

Feliú Jr., Sebastian

Felner, Israel

Fenu, Luigi

Fereiduni, Eskandar

Ferella, Francesco

Fernandes, Fábio

Fernández González, Daniel

Fernández, Juan Pedro

Fernández-Canteli, Alfonso

Fernandez-Vidal, Severo Raul

Ferrario, Mauro

Fidel, Salas Vicente

Fiedler, Thomas

Fiedorowicz, Henryk

Figueira, Rita

Figueroa, Raul

Fihey, Jean-Luc 
Filippov, Andrey

Fini, Stefano

Fischer, Claas

Fischer, Franz Dieter

Flege, Stefan

Fleury, Eric

Florczyk, Stephen

Florea, Corneliu

Florian Baron, Camilo

Flox, Cristina

Follansbee, Paul

Fontanari, Vigilio

Forbes, Liza

Fornaini, Carlo

Fornell, Jordina

Forouzan, Farnoosh

Forsey, Alexander N.

Fousova, Michaela

Fox-Rabinovich, German

Fracchia, Elisa

Fragassa, Cristiano

Franco, Francesco Di

Fraternali, Fernando

Frattini, Domenico

Freitas, Manuel José

Fricke, Wolfgang

Friedrich, Bernd

Fruchart, Daniel

Frydrych, Karol

Fuchs-Godec, Regina

Fuente, Daniel De La

Fujita, Takeshi

Fujiwara, Kozo

Fukumoto, Masahiro

Fulvio, Pasquale F.

Furushima, Tsuyoshi

Fuso, Francesco

Futáš, Peter

Fydrych, Dariusz

Gaffet, Eric

Gagliardi, Francesco

Gain, Asit Kumar

Gaitzsch, Uwe

Gajdzik, Bożena

Gajewski, Marcin

Galatolo, Roberto

Galietti, Umberto

Gallego, Antolino

Gallo, Pasquale

Galvão, Ivan

Gammer, Christoph

Gamsjäger, Ernst
Gangopadhyay, A. K.

Gangwar, Kapil

Ganvir, Ashish

Gao, Xu

Garbarz, Bogdan

Garbiec, Dariusz

Garces, Gerardo

García Diez, Ana

Garcia, C. Issac

Garcia-Escorial, Asuncion

Garcia-Mateo, Carlos

García-Moreno, Olga

Gariboldi, Elisabetta

Gaudry, Émilie

Gavrus, Adinel

Gawel, Tomasz

Gawlik, Józef

Gawronska, Elzbieta

Gedeon, Ondrej

Gee, Mark

Geijselaers, Hubert J.M.

Genau, Amber

Gentile, Marialuisa

Georgiou, Emmanuel P.

Gerbeth, Gunter

Gharam, Ahmed

Ghassemali, Ehsan

Gheorghe, Dan

Gheribi, Aimen Gheribi

Ghidelli, Matteo

Ghiuta, Ioana

Ghorbani, Mohammad

Ghosh, Monoj

Ghyngazov, Sergey Anatolievich

Giardini, Claudio

Giasin, Khaled

Gierl-Mayer, Christian

Gies, Soeren

Gigante, Giovanni E.

Gil, Javier

Gill, Richie H.S.

Gillan, Edward G.

Gilles, Ralph

Giorleo, Luca

Girish, Rughoobur

Girolami, Marco

Girot, Franck André

Giubileo, Filippo

Gjokas, Margaritis

Glaser, Björn

Glazov, Alexey L.

Gloc, Michał 
Gloria, Antonio

Glowacki, Miroslaw

Gnanasekaran, Karthikeyan

Gobber, Federico Simone

Golak, Sławomir

Goldbaum, Dina

Goldberg, Margarita A.

Goldmann, Daniel

Golgovici, Florentina

Gómez de Salazar Caso de los Cobos, José

María

Gómez, Xabier

Gómez-Parra, Álvaro

González, Beatriz

González, Haizea

González, Sergio

González-Doncel, Gaspar

Gonzalez-Gutierrez, Joamin

González-Rovira, Leandro

Goo, Byeong-Choon

Gorjan, Lovro

Górka, Jacek

Górny, Marcin

Gorunov, Andrey Igorevich

Goulas, Constantinos

Grabowski, Marcin

Graciani, Enrique

Graf, Marcel

Grajcar, Adam

Gramlich, Alexander

Gräning, Tim

Grasso, Marzio

Grasso, Salvatore

Grau, Gerd

Gravel, Edmond

Gray, Veronica

Greer, Andrew I. M.

Gric, Tatjana

Grivel, Jean-Claude

Grosdidier, Thierry

Grum, Janez

Grund, Thomas

Grunwald, Ruediger

Gruttadauria, Andrea

Gryc, Karel

Grydin, Olexandr

Grzegorczyk, Barbara

Grzesiak, Dariusz

Guarino, Stefano

Guastoni, Alessandro

Gucwa, Marek

Gueorguiev, G.K.
Guerra Rosa, Luis

Guerrero-Vaca, Guillermo

Guglielmino, Eugenio

Guillemot, Gildas

Gumienny, Grzegorz

Günther, Karsten

Gupta, Aniruddha

Gurau, Carmela

Gutierrez, Miguél Ángel

Guzanová, Anna

Gwalani, Bharat

Ha, Ngoc San

Habashi, Fathi

Habrat, Witold

HADDAG, Badis

Hadji, Ali

Haelsig, Andre

Hagenah, Hinnerk

Haghdadi, Nima

Hakimi, Orly

Hallberg, Håkan

Hallstedt, Bengt

Hamad, Kotiba

Hamada, Shigeru

HAMDI, Hédi

Hameed, Saqib

Hamilton, B. Carter

Hamilton, Carter

Hampton, Jennifer

Hampton, Michael

Han, Ke

Han, Yiwei

Hangai, Yoshihiko

Hänninen, Hannu

Hannula, Pyry-Mikko

Hansen, Klaus Schütt

Haque, Rezwanul

Harder, Ross

Harhash, Mohamed

Harničárová, Marta

Harrison, Noel

Härtel, Markus

Härtel, Sebastian

Hartl, Christoph

Hartmaier, Alexander

Hartwig, K.T.

Hasanian, Mostafa

Hassanieh, Amirhossein

Hassanin, Hany

Hatada, Ruriko

Hattori, Tomokazu

Haubrich, Jan 
Hawk, Jeffrey

Hawryluk, Marek

Hayakawa, Tohru

Hayat, Muhammad

Hazar, Selcuk

Hegab, Hussien

Hegedüs, Csaba

Heimann, Robert

Heise, Bettina

Heise, Rainer

Helle, Mikko

Hellsten, Niko

Hemery, Samuel

Henaff, Gibert

Hermann, Andreas

Hermawan, Hendra

Hernández Saz, Jesús

Hernández-Nava, Everth

Hernando, Juan Carlos

Herper, Heike

Hertele, Stijn

Hidalgo Manrique, Paloma

Hidalgo, Javier

Hildebrand, Joerg

Hiremath, Nitilaksha

Hirohata, Mikihito

Hitzler, Leonhard

Ho, Hsing-Jung

Hobosyan, Mkhitar

Hoch, Constantin

Hochrainer, Thomas

Hodulova, Erika

Hof, Lucas A.

Hohenwarter, Anton

Hojná, Anna

Hojo, Tomohiko

Hokamoto, Kazuyuki

Holappa, Lauri

Holdengreber, Eldad

Holm, Elizabeth

Holynska, Malgorzata

Hong, Min

Hong, Minghui

Hong, Seokmoo

Hong, Soon-Jik

Hong, Sung Hwan

Hong, Sung-Tae

Honus, Stanislav

Hooli, Paavo

Höppel, Heinz Werner

Horky, Jelena

Horodek, Paweł
Hosseini, Vahid A.

Hosseinpour, Saman

Hossfeld, Max

Housecroft, Catherine

Hovanski, Yuri

Hryniewicz, Tadeusz

$\mathrm{Hu}$, Jong Wan

Hug, Eric

Hulme-Smith, Christopher Neil

Huot, Jacques

Hur, Dohaeng

Hurley, Mike

Hurst, Roger

Hütter, Geralf

Hvizdoš, Pavol

Hwang, Seong Sik

Hyk, Wojciech

Ijiri, Masataka

Ilea, Petru

Iljkić, Dario

Iluk, Artur

Im, Kwang-Hee

Imperatore, Stefania

Inoue, Hirofumi

Ionescu, Mihail

Iqbal, Asif

Irani, Missam

Iribarren, José Ignacio

Isanaka, Sriram Praneeth

Isasti, Nerea

Ismail, Umair

Ito, Kiyohiro

Ito, Mikio

Ito, Tsutomu

Ivanić, Ivana

Ivanisenko, Julia

Ivanov, K.V.

Iwamoto, Takeshi

Iwaszko, Jozef

Jabraoui, Hicham

Jackson, Mark

Jackson, Martin

Jacob, Isaac

Jahromi, Fazel G.

Jakovljević, Suzana

Jakumeit, Juergen

James, Sagil

Jamrozik, Wojciech

Jang, Changheui

Jang, Heejin

Jankauskas, Vytenis

Janovec, Jozef 


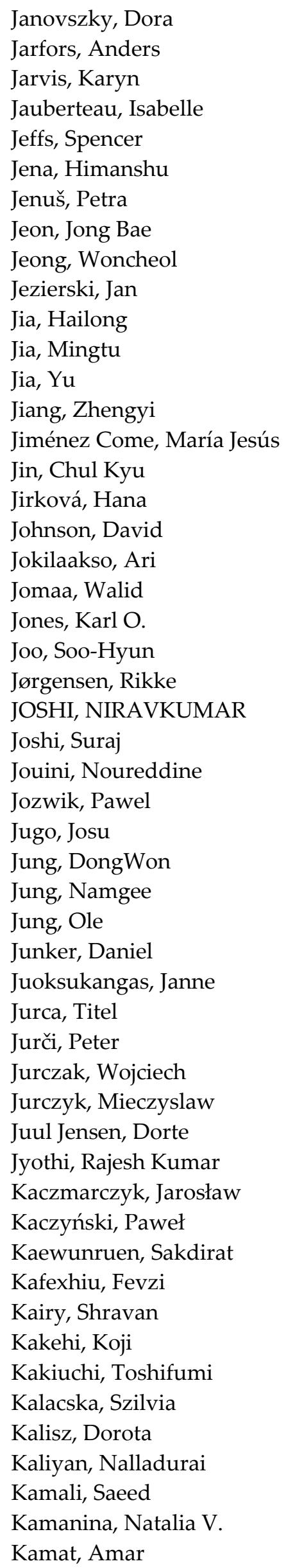

\author{
Kamiński, Marcin \\ Kamiya, Osamu \\ Kanari, Ndue \\ Kaneko, Kenji \\ Kaneko, Yoshihisa \\ Kang, Chung Gil \\ Kang, Jun-Yun \\ Kang, Nam-Hyun \\ Kannan, Manigandan \\ Kaplan, Valery \\ Kapłonek, Wojciech \\ Kappagantula, Keerti \\ Karakalas, Anargyros \\ Karbowniczek, Joanna \\ Karczewski, Krzysztof \\ Karg, Michael Cornelius Hermann \\ Karjalainen, L. Pentti \\ Karnati, Sreekar \\ Karolczuk, Aleksander \\ Karolus, Malgorzata \\ Kashani, Hamzeh \\ Kasińska, Justyna \\ Katarivas Levy, Galit \\ Katerski, Atanas \\ Katz, Jonathan I. \\ Kaufman, Michael J. \\ Kawai, Toshiyuki \\ Kawakita, Hidetaka \\ KAWALEC, M \\ Kawalec, Magdalena \\ Kaynak, Yusuf \\ Kazansky, Leonid \\ Kazek-Kęsik, Alicja \\ Kechagias, John \\ Keckes, Jozef \\ Keist, Jayme S. \\ Keller, Clément \\ Kenzari, Samuel \\ Keppert, Martin \\ Kern, Frank \\ Kessler, Sylvia \\ Ketov, Sergey \\ Khameneifar, Farbod \\ Khamkhash, Aibyek \\ Khan, Mohammad Ashraf \\ Khanal, Rabi \\ Kharin, Viktor \\ Khoddam, Shahin \\ Khotinov, Vladislav A. \\ Khrustalyov, Anton \\ Kiełbus, Andrzej \\ Kik, Tomasz
}


Kikuchi, Hiroaki

Kim, Chan-Jung

Kim, Cheolhee

Kim, Hyunjung

Kim, Insoo

Kim, Jangho

Kim, Jeong Tae

Kim, Ji Hoon

Kim, JungGu

Kim, Keun-Soo

Kim, Kyungmok

Kim, Kyung-Taek

Kim, Man-Hoe

Kim, Moon-Jo

Kim, Seon-Jin

Kim, Soo Young

Kim, Su-Hyeon

Kim, Sun-Joong

Kim, Young Suk

Kim, Young-Min

Kimura, Akihiko

Kiryukhantsev-Korneev, Philipp

Vladimirovich

Kiss, Imre

Kitagawa, Jiro

Kleijnen, Rob

Kleimt, Bernd

Klein, Marcus

Klemettinen, Lassi

Klemm, Richard

Kloc, Lubos

Klöcker, Helmut

Kloeden, Burghardt

Klose, Christian

Klusemann, Benjamin

Kmita, Angelika

Knowles, Alexander

Knyazeva, Marina

Ko, Won-Seok

Ko, Yong-Ho

Kobayashi, Makiko

Koblischka, Michael R.

Kochmańska, Agnieszka

Kochmański, Paweł

Koibuchi, Hiroshi

Kojima, Kenichi

Kölbl, Nathalie

Kollár, Peter

Komarov, Sergey

Komasa, Satoshi

Kombaiah, Boopathy

Konat, Łukasz
Konda Gokuldoss, Prashanth

Kondracki, Marcin

Konovalov, Sergey

Konstanciak, Anna

Konstanty, Janusz

Kontoni, Denise-Penelope N.

Konyukhov, Andrey

Koohbor, Behrad

Koohbor, Behrad Behrad

Kopeček, Jaromír

Kopyściański, Mateusz

Koric, Seid

Korlos, Apostolos

Korneva, Anna

Kornienko, Elena

Korosteleva, Elena N.

Korsunsky, Alexander M.

Korzeniewska, Ewa

Košak, Aljoša

Kosec, Prof.dr. Borut

Kostolný, Igor

Kostryzhev, Andrii

Kosturek, Robert

Koteš, Peter

Kotov, Anton

Koudelka, Petr

Kourousis, Kyriakos

Koutny, Daniel

Koutzarova, Tatyana

Kovacik, Jaroslav

Kovács, Zsolt

Kovarik, Ondrej

Kowalczuk, Przemyslaw B.

Kowalska, Ewa

Krajewski, Witold

Král, Petr

Krawczyk, Jacek

Kreislova, Katerina

Krejcar, Ondrej

Krenický, Tibor

Křivý, Vit

Król, Mariusz

Królczyk, Grzegorz

Królicka, Aleksandra

Kroll, Peter

Krouse, Donal

Krylova, Alla Yu.

Krymsky, Valeriy V.

Krzanowski, James

Krzanowski, James E.

Książek, Marzanna

Ktena, Aphrodite 
$\mathrm{Ku}$, Tae-Wan

Kubisztal, Julian

Kubit, Andrzej

Kučerová, Ludmila

Kuczmaszewski, Jozef

Kuhn, Bernd

Kujawa, Joanna

Kujawski, Daniel

Kukla, Christian

Kulagin, Roman

Kulka, Michal

Kumar, Dhananjay

Kümmel, Frank

Kunčická, Lenka

Kundin, Julia

Kunetsov, Vladimir

Kuntz, Matthias

Kurek, Andrzej

Kurlyandskaya, Galina V.

Kurylo, Piotr

Kurzydłowski, Krzysztof J.

Kusinski, Jan P.

Kustov, Leonid

Kustov, Leonid M.

Kut, Stanisław

Kuźnicka, Bogumiła

Kvačkaj, Tibor

Kvapilova, Marie

Kwan, Charles

Kwaśniak, Piotr

Kwolek, Przemysław

Kwon, Eui Pyo

Kwon, Hansang

Kwon, Nam Hee

Kwon, Tae-Yub

Kyratsis, Panagiotis

La Magna, Antonino

Lacalle, Roberto

Lacoste, Eric

Laheurte, Pascal

Laiarinandrasana, Lucien

Lambiase, Francesco

Lamikiz, Aitzol

Lampakis, Dimitrios

Lampke, Thomas

Lancaster, Robert

Landowski, Michał

Langer, Kristina

Lanjewar, Harishchandra

Lapin, Juraj

Laptev, Alexander

Larkiola, Jari
Larouche, Daniel

Larsson, Ragnar

Lashgari, Hamid R.

Łatka, Leszek

Lattanzi, Lucia

Laureti, Sara

Lavvafi, Hossein

Lazarescu, Lucian

Lazurenko, Daria V

Le, Chau

Leal, Rui

Lebyodkin, Mikhaïl A.

Lecler, Sylvain

Lecomte, Jean-Sebastien

Lee, Byeongchan

Lee, Chang-Whan

Lee, Geuntak

Lee, H. W.

Lee, Jae Myung

Lee, Jeonghoon

Lee, Jinwoo

Lee, Jongbeom

Lee, Jongsup

Lee, Jung-Moo

Lee, Kee Sung

Lee, Myoung Gyu

Lee, Sang-Kon

Lee, Seok-Jae

Lee, Wookjin

Lee, Young-Kook

Lee, Yousub

Lehmhus, Dirk

Leineweber, Andreas

Leitner, Martin

Leiviskä, Kauko

Lekakh, Simon

Leliaert, Jonathan

Lemu, Hirpa G.

Lengauer, Walter

Lennartsson, Andreas

Leonidas, Palilis

Leonowicz, Marcin

Lepicka, Magdalena

Lequien, Florence

Lesiuk, Grzegorz

Lessard, Benoit

Lesselier, Dominique

Lesz, Sabina

Leszczyńska-Madej, Beata

Leszczyńska-Sejda, Katarzyna

Letcher, Todd

Lewis, Gladius 
Lgaz, Hassane

Li, Jiehua

Li, Zhiming

Lian, Junhe

Lichý, Petr

Lienert, Ulrich

Limmer, Krista R.

Limodin, Nathalie

Lindemann, Inge

Lindner, Thomas

Lindsay, Robert

Lipiński, Tomasz

Lipski, Adam

Lisiecka-Graca, Paulina

Lisiecki, Aleksander

Liu, Jun

Llavori, Iñigo

Lloveras, Pol

Lo Conte, Antonietta

Lo Frano, Rosa

Löbel, Martin

Lobo, Rui F. M.

Loginova, Irina

López de Lacalle, Luis Norberto

López, Beatriz

Lopez, Felix A.

Lopez, Gabriel Alejandro

Lopez-Crespo, Pablo

Lorenzi, Sergio

Lorenzo, Miguel

Lorusso, Massimo

Löser, Wolfgang

Łosiewicz, Bożena

Louhenkilpi, Seppo

Louzguine, Dmitri V.

Lüchinger, Marco

Luis, Barrales Mora

Lukáč, Pavel

Lukács, János

Lukaszkowicz, Krzysztof

Lunt, Alexander

Luo, Liang

Luo, Quanshun

Luong, Dũng (Dzung) Dinh

Lusquiños, Fernando

Luukkonen, Tero

Ly, Ramatou

Łyszkowski, Radosław

Ma, Xiang

Ma, Yan

Maamoun, Ahmed

Machno, Magdalena
Madison, Jonathan

Magdysyuk, Oxana

Maia, Frederico

Maier, Petra

Maietta, Saverio

Maina, Martin Ruthandi

Maiorino, Angelo

Maj, Michał

Majerníková, Janka

Majlinger, Kornel

Majstorovic, Vidosav D.

Makabe, Chobin

Makarov, Dmitriy V.

Makineni, Surendra Kumar

Maksoud, Talal

Malakhov, Dmitri

Maliaris, Georgios

Mandal, Paranjayee

Mandolfino, Chiara

Manière, Charles

Mankey, Gary

Manojlović, Vaso

Manolakos, Dimitrios

Manuel, Pascual Guillamón

Manzoni, Anna

Mapelli, Carlo

Marceau, Ross K. W.

Marchese, Giulio

Marcinauskas, Liutauras

Marcisz, Jaroslaw

Marcisz, Jarosław

Mărginean, Gabriela

María Rodríguez-Ibabe, José

Marinelli, Gianrocco

Marinkovic, Dragan

Markopoulos, Angelos

Marohnic, Tea

Marônek, Milan

Marsavina, Liviu

Marshall, Jessica

Marszalek, Marta

Marthinsen, Knut

Martin, Sylvain

Martín-Crespo, Tomás

Martinelli, Enzo

Martinez Khramer, Daniel

Martínez-Huerta, María Victoria

Martínez-Lillo, José

Martinez-Martinez, Diego

Martini, Carla

Martins Amaro, Ana Paula Betencourt

Martins, João M. P. 
Maruda, Radosław W.

Marx, Michael

Masatoshi, Nishi

Mastorakos, Ioannis

Masuda, Hiroshi

Masuda, Yoshitake

Matanović, Ivana

Mateo, Antonio

Materer, Nicholas F.

Matino, Ismael

Matsukuma, Hiraku

Matsuno, Takashi

Matsuo, Koichi

Matsushima, Yuta

Matsushita, Masafumi

Matsushita, Taishi

Matsuyama, Tatsushi

Matteis, Paolo

Matula, Grzegorz

Matykina, Endzhe

Matys, Jacek

Matz, Alexander Martin

Mauder, Tomáš

Maurel, Vincent

Maurice, David

Maximov, Maxim

Mayr, Peter

Mayuet-Ares, Pedro

Mazalski, Piotr

Mazur, E. A.

Mazur, Igor

Mazzù, Angelo

Mazzucato, Federico

McDonald, Robbie

McFadden, Shaun

McLean, Alexander

Mcnelley, Terry R.

Medyński, Daniel

Meenashisundaram, Ganesh Kumar

Mehner, Thomas

Mehrpouya, Mehrshad

Mendes, Ricardo

Meneghetti, Giovanni

Menezes, Pradeep

Menzemer, Craig

Merkel, Markus

Merklein, Marion

Mertens, Anne

Meshi, Louisa

Mészàros, Istvàn

Miani, Fabio

Michalska-Domańska, Marta
Michelic, Susanne

Miculescu, Florin

Miculescu, Marian

Mierzejewska, Żaneta Anna

Miguélez, M. Henar

Mikhaylovskaya, Anastasia

Mikhlin, Yuri

Miki, Hajime

Miklaszewski, Andrzej

Mikolajczyk, Tadeusz

Milčius, Darius

Mileiko, Sergei T.

Milenin, Andrij

Milinovic, Andrijana

Milke, Ralf

Milkovič, Ondrej

Miłkowska-Piszczek, Katarzyna

Milosevic, Milorad

Minařík, Antonín

Mingler, Bernhard

Mirihanage, W. U.

Mironov, S.

Mironov, Sergey

Mirzadeh, Hamed

Mishin, Oleg

Mistewicz, Krystian

Mitchell, Alec

Mitoraj-Królikowska, Marzena

Mitsui, Yoshifuru

Mittelman, Brigit

Mityushev, Valdimir

Miyake, Masao

Miyamoto, Hiroyuki

Miyazaki, Toshiki

Mock, Clara

Modigell, Michael

Moeini, Ghazal

Mogeritsch, Johann

Mogucheva, Anna

Mohammadiarani, Hossein

MOHAMMED, ABDUL SAMAD

Mohanta, Antaryami

Mohanty, Sankhya

Mohles, Volker

Mohrbacher, Hardy

Mohtadi Bonab, Mohammad Ali

Molnár, Vieroslav

Molnar, Viktor

Mombelli, Davide

Momeni, Mojtaba

Monchoux, Jean-Philippe

Monetta, Tullio 


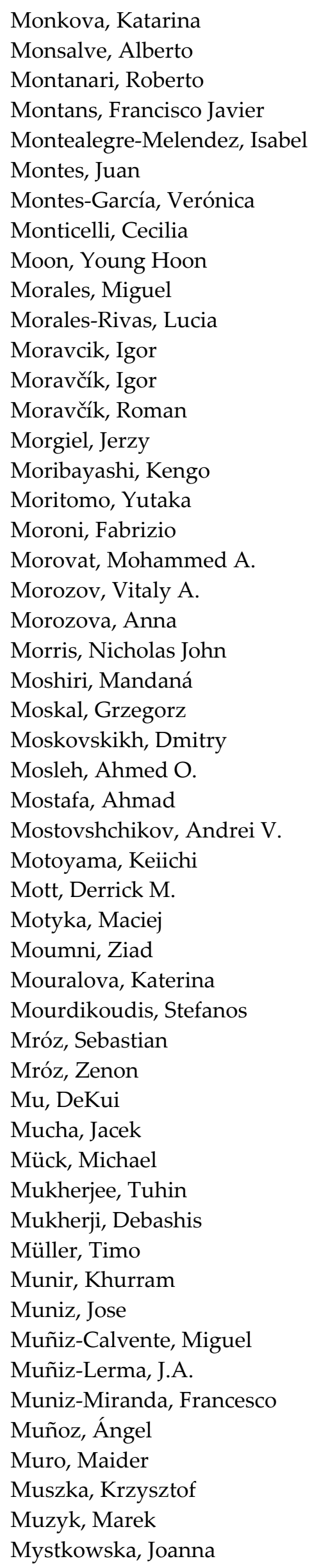

Nabeel, Muhammad

Nadolny, Krzysztof

Nagaoka, Toru

Naher, Sumsun

Nakagawa, Yuki

Nakahata, Kazuyuki

Nalivaiko, Anton Yu

Nambu, Koichiro

Naplocha, Krzysztof

Narciso, Javier

Narita, Hirokazu

Naser, M. Z.

Navarro Pintado, Carlos

Nawani, Pranav

Nazarov, Andrej P.

Nazarov, Denis V.

Nazir, Aamer

Nebreda, Jaime Lazaro

Nejbert, Krzysztof

Nemchinova, Nina

Nene, Saurabh

Neslušan, Miroslav

Neto, Diogo M.

Nguyen Minh, Tuan

Nguyen, Van Khanh

Nguyen-Manh, Duc

$\mathrm{Ni}$, Peiyuan

Nicoara, Mircea

Niesłony, Piotr

Niessen, Frank

Nieves, Javier

Nikhare, Chetan

Nikumb, Suwas

Ning, Jinqiang

Nishikawa, Arthur

Nishimoto, Satoshi

Nisticò, Roberto

Njock Bayock, Francois

Nobile, Lucio

Nobile, Riccardo

Normand, Bernard

Nosal, Przemysław

Nothdurft, Sarah

Novak, Pavel

Novák, Pavel

Novelli, Marc

Nowacki, Jerzy

Nowak, Wojciech Jerzy

Nowak, Zdzisław

Oancea, Gheorghe

Obraztsov, Alexander

Obrosov, Aleksei 
Obuchowicz, Andrzej

Oezkaya, Ekrem

Ogawa, Fumio

Oh, Jonghyun

Oh, Min-wook

Okitsu, Yoshitaka

Okoshi, Masayuki

Okrajni, Jerzy

Oksiuta, Zbigniew

Okunkova, Anna A.

Olbricht, Jürgen

Oliveira, João Pedro

Oliveira, Marta

Olmi, Giorgio

Olofinjana, Ayodele

Olsson, Rickard

Olszówka-Myalska, Anita

Omar, Ahmed

Omar, Faye

Omrani, Emad

Onghena, Bieke

Ono, Kanji

Optasanu, Virgil

Orbulov, Imre

Orlov, Dmytro

Orlov, V. M.

Örnek, Cem

Ortega, José Marcos

Oskouei, Reza H.

Osmanlic, Fuad

Österreicher, Johannes Albert

Osuch, Piotr

Owolabi, Gbadebo

Ozerov, Maxim

Păcurar, Răzvan

Pagan, Darren

Pahlevani, Farshid

Paiva, Ana Paula

Palimaka, Piotr

Palkowski, Heinz

Pallaspuro, Sakari

Palumbo, Davide

Panagopoulos, C. N.

Pandey, Sudip

Panigrahi, Mrutyunjay

Panowicz, Robert

Pantazopoulos, George A.

Panteghini, Andrea

Paolone, Annalisa

Papaefthymiou, Spyros

Papanikolaou, Michail

Papanikolaou, Stefanos
Parfenov, Evgeny

Parian, Mehdi

Park, Deok-Yong

Park, Hong-Seok

Park, Joon Sik

Park, Jun-Sang

Park, Kwangsuk

Park, Nokeun

Park, Seong Jin

Park, Soo-Jin

Parker, Jonathan

Parsa, Alireza B.

Partovi-Nia, Rachel

Pascoe, John-Alan

Pasebani, Somayeh

Pashos, George

Pastorek, Filip

Patel, Vikas

Pater, Zbigniew

Pathakoti, Kavitha

Patil, Sachin

Patil, Sandeep

Patisson, Fabrice

Patterson, Albert

Patterson, Albert E.

Pattini, Francesco

Paul, Henryk

Paul, Shiladitya

Paulin, Irena

Pawlus, Paweł

Pearce, Joshua

Pedicini, Rolando

Pedraza, Fernando

Pedrini, Giancarlo

Pedzich, Zbigniew

Peixinho, Nuno Ricardo Maia

Pejkowski, Łukasz

Pelletier, J.M.

Pellizzari, Massimo

Penz, Florian Markus

Pepelnjak, Tomaz

Perdahcioglu, Semih

Pereira Falcon, Juan Carlos

Pereira Gonçalves, António

Pereira, António

Pereira, Octavio

Peretyagin, Pavel

Pérez Rodríguez, Sara

Pérez-Soriano, Eva M.

Pérez-Tello, Manuel

Périgo, Élio Alberto

Perrella, Michele 
Peter, Ildiko

Petković, Bojana

Petrescu, Lucian

Petrič, Mitja

Petrillo, Antonella

Petrov, Peter

Petrov, Roumen

Petru, Jana

Petry, Winfried

Petryshynets, Ivan

Pettignano, Alberto

Pettinari-Sturmel, Florence

Peura, Pasi

Peyre, Patrice

Pezzato, Luca

Phuah, Xin Li

Picard, Donald

Pickering, Ed

Piedade, Ana Paula

Pierron, Olivier

Pietranik, Anna

Pietrusiak, Damian

Pilarczyk, Wirginia

Pimenov, Danil Yu

Pineda, Eloi

Pingitore, Nicholas

Pistidda, Claudio

Pistorius, P. Chris

Piszczek, Piotr

Plott, Jeff

Plucknett, Kevin

Po, Giacomo

Pogatscher, Stefan

Pokusová, Marcela

Polanski, Marek

Polatidis, Efthymios

Pollmann, Katrin

Polozov, Igor

Pons, Dirk

Ponticelli, Gennaro Salvatore

Poon, Joseph

Poorteman, Marc

Pop, Mihai Alin

Popa, Florin

Popescu, Andrei

Pötschke, Johannes

Pou, Juan

Poullain, Philippe

Pourret, Olivier

Poyato, Rosalía

Pozdniakov, Andrey

Poznak, Alex
Prabakaran, Mayakrishnan

Prados-Frutos, Juan-Carlos

Prados-Privado, María

Pramanik, Brahmananda

Pramudita, Jonas A.

Prasad, Anil

Prasadh, Somasundaram

Prashanth, Konda Gokuldoss

Prates, Pedro

Presz, Wojciech

Priel, Elad

Prinz, Katharina

Prisco, Umberto

Prosek, Tomas

Prošek, Tomáš

Prosviryakov, Alexey S.

Pruncu, Catalin

Prusov, Evgeny

Pryamikov, Andrey

Przekora, Agata

Przestacki, Damian

Psuj, Grzegorz

Psyllaki, Pandora

Puchi-Cabrera, Eli Saul

Puga, Hélder

Pushilina, Natalia

Pustovoytov, Denis

Puszka, Andrzej

Pyszko, René

Pyun, Young Sik

Qi, Zhiyuan

Quémerais, Bernadette

Rachik, Mohamed

Racz, Gabriel

Radtke, Aleksandra

Radu, Dorin

Rǎducanu, Doina

Radwański, Krzysztof

Ragab, Khaled

Rahman Rashid, Rizwan Abdul

Raith, Stefan

Rajala, Pauliina

Rajic, Nik

Rakhmonov, Jovid

Ramazani, Ali

Ramos, Ana

Ranganathan, Narayanaswami

Ranitović, Milisav

Rankin, Rees B.

Ranzi, Eliseo

Rao, Jeff

Raoelison, Rija Nirina 
Rashidi, Masoud

Raudensky, Miroslav

Raush, Jonathan

Ravindra (Ravi), Nuggehalli M.

Ravindran, Ravi

Rechendorff, Kristian

Rege, Kristen

Regel-Rosocka, Magdalena

Regev, Michael

Reid, Andrew C.E.

Reis, Anabela

Rescalvo, Francisco

Rešković, Stoja

Rezaei Rad, Aryan

Rezvani, Ehsan

Rhamdhani, M Akbar

Riahi, Reza

Ribeiro, Carlos A. Silva

Ribeiro, João

Ribeiro, José Baranda

Riccio, Aniello

Richert, Maria

Richeton, Thiebaud

Richetta, Maria

Riedl, Helmut

Rigby, Sam

Ripamonti, Dario

Riva, Sephira

Riveiro, Antonio

Rivero, Pedro J.

Rivière-Lorphèvre, Edouard

Roa Rovira, Joan Josep

Robertson, David G.

ROCA, ANTONIO

Rodgers, Theron

Rodríguez Millán, Marcos

Rodriguez, Mario H.

Rodríguez, Olga

Roebuck, Bryan

Rogal, Łukasz

Rogala, Andrzej

Rogalski, Grzegorz

Rogovoy, Anatoly A.

Rogozhnikov, Denis A.

Rojas, Jose I.

Rokni, Michael Reza

Rokosz, Krzysztof

Roldán, Marcelo

Romanowicz, Pawel

Romanowicz, Paweł

Romero-Muñiz, Carlos

Ropital, François
Rosso, Mario

Rotella, Giovanna

Roth, Johannes

Rout, Bapin Kumar

Roy, Matthew

Rozumek, Dariusz

Rtimi, Sami

Rudolf, Rebeka

Ruello, Maria Letizia

Ruffino, Francesco

Ruiz-Cabello, Francisco Javier Montes

Ruoppolo, Giovanna

Russell, Alan M.

Russo Spena, Pasquale

Ruuska, Jari

Rynkovskaya, Marina

Rzelewska-Piekut, Martyna

Sabirov, Denis

Sabirov, Ilchat

Saboori, Abdollah

Saeed, Adil

Safari, Mehdi

Saffari Pour, Mohsen

Saito, Noritaka

Saito, Takeshi

Saito, Tetsuji

Sajadifar, Seyed Vahid

Sakharova, Nataliya

Sakon, Takuo

Sałaciński, Tedeusz

Salama, Walid

Sales, Rita De Cássia Mendonça

Salguero, Jorge

Salim, Hani

Salmi, Mika

Salminen, Antti

Saltas, Vassilis

Salvati, Enrico

Salvia, Michelle

Samadi-Dooki, Aref

Samborski, Sylwester

Samei, Javad

Samek, Ludovic

Samoila, Cornel

Samouhos, Michail

Samuha, Shmuel

Sánchez Egea, Antonio

Sánchez Egea, Antonio J.

Sánchez, Antonio

Sanchez, Jose M.

Sand, Anders

Sander, Manuela 
Sangiovanni, Davide

Santamaria, Monica

Santecchia, Eleonora

Santoni, Andrea

Santos, Rafael

Santos, Rafael M.

Sapozhnikov, Konstantin

Sarac, Baran

Sarker, Dyuti

Sarraf, Iman Sari

Sastoque Pinilla, Leonardo

Saternus, Mariola

Sato, Eiichi

Savkovic, Borislav

Sawicki, Jacek

Saxen, Henrik

Scendo, Mieczyslaw

Schajer, Gary

Schenk, Johannes

Schenk, Thomas

Schindler, Ivo

Schlesinger, Mark

Schmauder, Siegfried

Schmidova, Eva

Schmidtchen, Matthias

Schwarm, Samuel

Schwarz, Philip

Scott, Colin

Scribante, Andrea

Scutelnicu, Elena

Secco, Luciano

Sedelnikova, Mariya B.

Segreto, Tiziana

Seifan, Mostafa

Seiler, Philipp

Seiner, Hanuš

Seko, Noriaki

Semaltianos, Nikolaos G.

Semboshi, Satoshi

Semenova, Irina P.

Semih, Ener

Senderowski, Cezary

Senk, Dieter

Senos, Ana Maria De Oliveira Rocha

Sepe, Raffaele

Sepúlveda Ferrer, Ranier Enrique

Serban, Nicolae

Sergejev, Fjodor

Serna, Sergio

Serrano, Ricardo Fernández

Sertucha, Jon

Šetina Batič, Barbara
Setti, Dinesh

Sglavo, Vincenzo M.

Shaha, Sugrib

Shaha, Sugrib Kumar

Shalaby, Mohamed

Shalchi Amirkhiz, Babak

Shams, Alireza

Sharkeev, Yurii

Shayesteh Moghaddam, Narges

Shchetinin, Igor

Shchyglo, Oleg

Shevchenko, Sergey

Shi, Zhusheng

Shibuta, Yasushi

Shiers, Denis

Shimamura, Yoshinobu

Shimoi, Norihiro

SHIMONO, Masato

Shin, Keesam

Shiomi, Masanori

Shirai, Kouun

Shiraiwa, Takayuki

Shiratori, Tomomi

Shishin, Denis

Shishkin, Andrei

Shokrani, Alborz

Shunmugasamy, Vasanth C.

Sidor, Jurij J.

Silhanek, Alejandro V.

Siller, Hector

Silva, Francisco

Silva, Francisco J. G.

Silva, M. Beatriz

Silva, Maria Beatriz

Silvello, Alessio

Simka, Wojciech

Simm, Stefan

Simões, Sónia

Simon, Franz-Georg

Simoncini, Michela

Simonelli, Marco

Sinebryukhov, Sergey Leonidovich

Sing, Swee Leong

Singh, Alok

Singh, Deepak Pratap

Siredey-Schwaller, N.

Siska, Filip

Sismanis, Panagiotis

Sitek, R.

Siwak, Piotr

Skoczylas, Agnieszka

Skrbek, Bretislav 
Skrotzki, Birgit

Slater, Carl

Slifka, Andrew J.

Slobodyan, Mikhail

Slota, Ján

Ślusarczyk, Łukasz

Smaga, Marek

Smolina, Irina

Smyrak, Beata

Sniezek, Lucjan

Sobianowska-Turek, Agnieszka

Socha, Ladislav

Socha, Tomasz

Sohn, Seok Su

Sokić, Miroslav D.

Solanki, Raj

Solano, Wilberth

Solá-Ruíz, María F.

Solimani, Ali

Solonin, Alexey

Sommerseth, Camilla

Somsen, Christoph

Song, Miao

Sonsteby, Henrik Hovde

Sopicka-Lizer, Małgorzata

Sorgente, Donato

Sotov, Anton

Soucy, Gervais

Souto, Ricardo Manuel

Soyama, Hitoshi

Sozańska, Maria

Speicher, Magdalena

Spendier, Kathrin

Spieß, Lothar

Spigarelli, Stefano

Spiller, Erik

Spišák, Emil

Spooren, Jeroen

Srnec Novak, Jelena

Sroka, Marek

Staat, Manfred

Stachiv, Ivo

Stachurski, Z.H.

Staicu, Lucian

Stanford, Nicole

Starke, Peter

Starosta, Wojciech

Starostenkov, Mikhail

Starov, Victor

Stavropoulos, Panagiotis

Stawarz, Marcin

Steenari, Britt-Marie
Steer, Julian

Stefanik, Andrzej

Stefanoiu, Radu

Stefanou, George

Stein, Nicolas

Steinberg, Simon

Steiner, Matthew A.

Steinlechner, Stefan

Stepanov, Nikita

Stępniowski, Wojciech

Stergioudi, Fani

Stern, Adin

Stiharu, Ion

Stoia, Dan Ioan

Stopic, Srecko

Stopić, Srećko

Storti, Enrico

Stoulil, Jan

Stoyan, Dietrich

Stradomski, Grzegorz

Straumal, Boris B.

Straže, Aleš

Strnadel, Bohumír

Strozzi, Antonio

Strunz, Pavel

Strzemiecka, Beata

Stückler, Martin

Stuhr, Uwe

Šturm, Roman

$\mathrm{Su}$, Yingchao

Suárez, Oscar Marcelo

Suder, Wojciech

Sugimoto, Koh-ichi

Suh, Jin-Yoo

Sulasalmi, Petri

Sulitka, Matej

Sun, Y.F.

Sun, Yongle

Sung, Jaeyong

Suñol, Joan-Josep

Surmeneva, Maria A.

Surowiak, Agnieszka

Surzhenkov, Andrei

Susan, Donald F.

Suzuki, Ryosuke O.

Suzuki, Tomoya

Svec, Peter

Sveda, Maria

Svoboda, Jiri

Swiatkowska, Zaneta

Świercz, Rafał

Świerczyńska, Aleksandra 
Szala, Miroslaw

Szczotok, Agnieszka

Szczucka-Lasota, Bożena

Szewczyk, Roman

Szielasko, Klaus

Szkliniarz, Agnieszka

Szkodo, Marek

Szmytka, Fabien

Szwajka, Krzysztof

SZYBIŃSKI, BOGDAN

Szymczak, Tomasz

Tabelin, Carlito

Tada, Naoya

Tagirov, Boris R.

Takahashi, Kazuyuki

Takahashi, Keisuke

Takahashi, Koji

Takahashi, Masatoshi

Takahiro, Katsumi

Takaichi, Atsushi

Takasugi, Takayuki

Taler, Jan

Tallarico, Marco

Tamburrino, Francesco

Tamura, Koji

Tang, Tiger

Taninouchi, Yu-ki

Tanner, David

Tapoglou, Nikolaos

Tappa, Karthik

Tarasov, Sergei Yu

Tashiro, Shinichi

Taskinen, Pekka

Tatsumi, Hiroaki

Taupin, Vincent

Tavanti, Francesco

Tavares, S.M.O.

Taxiarchou, Maria

Tayebi-Khorami, Maedeh

Taylor, M.P.

Tcherdyntsev, Victor V.

Teghil, Roberto

Teixeira, Julien

Tekumalla, Sravya

Telegdi, Judit

Temprano, Israel

Terčelj, Milan

Testa, Gabriel

Testani, Claudio

Texier, Damien

Thackray, Richard

Thadhani, Naresh
Thai, Duc-Kien

Thapa, Dinesh

Thapa, Prem S.

Thapliyal, Vivek

Thom, Christopher A.

Thomä, Marco

Thuillier, Sandrine

Thuo, Martin

Thuvander, Mattias

Tic, Wilhelm Jan

Tielens, Frederik

Tiferet, Eitan

Timmel, Klaus

Timon, Rabczuk

Tirillo', Jacopo

Tkadleckova, Marketa

Toboła, Daniel

Tocci, Marialaura

Todoroki, Hidekazu

Tokumoto, Yuki

Tolnai, Domonkos

Tomáš, Miroslav

Tomasz, Durejko

Tomczak, Janusz

Tomiczek, Błażej

Tomków, Jacek

Tomkowski, Robert

Tomota, Yo

Tonelli, Domenica

Tonti, Andrea

Tora, Barbara

Toribio, Jesús

Torić, Neno

Torrens-Serra, Joan

Torrisi, Vanna

Torzewski, Janusz

Toth, Laszlo

Treutler, Kai

Tribe, Lorena

Tridello, Andrea

Trifiletti, Vanira

Troiani, Enrico

Trojanova, Zuzanka

Trotta, Gianluca

Truster, Timothy

Trybuła, Marcela E.

Trytek, Andrzej

Trzepiecinski, Tomasz

Tsakiridis, Petros E.

Tsao, Lung-Chuan

Tserpes, Konstantinos

Tsyganenko, Alexey A. 
Tuominen, Jari

Tuovinen, Olli

Turenne, Sylvain

Turner, Richard

Tussupzhanov, Aidyn

Twardowski, Paweł

Tylczak, Joseph

Ubertalli, Graziano

Udroiu, Razvan

Ueda, Mikito

Ueji, Rintaro

Uematsu, Y.

Uemori, Takeshi

Ueshima, Nobufumi

Ulewicz, Robert

Underwood, Olivia D.

Unruh, Daniel

Urbani, Maxence

Urbicain, Gorka

Urbikain, Gorka

Urieta, José

UTU, Ion-Dragos

Uzunlar, Erdal

Vairis, Achilles

Vakhrushev, Alexander

Valdemar Fernandes, José

Valeev, Dmitry

Valente, Robertt

Valentini, Renzo

Vallejo, Javier P.

Valvano, Stefano

Valvo, Paolo S.

Van Der Sluis, Olaf

van der Zwaag, Sybrand

Van Hullebusch, Eric

Van Renterghem, Wouter

Varga, Lajos

Varma, Shailendra Krishna

Varone, Alessandra

Vasconcelos, Helena Cristina

Vastola, Guglielmo

Vazquez Martinez, Juan Manuel

Vázquez Valeo, Jesús

Vedyagin, Aleksey A.

Velasco Lopez, Francisco Javier

Velasco-Ortega, Eugenio

Velkavrh, Igor

Venettacci, Simone

Venkata, Kiranmayi Abburi

Verdeja González, Luis Felipe

Verdeja, Luis Felipe

Vereshchaka, Alexey A.
Vergara Rodríguez, Diego

Vergara, Diego

Verleysen, Patricia

Vidal, Catarina

Vieira, Manuel

Vijayaraghavan, Venkatesh

Villa, Elena

Villa, Matteo

Vincze, Gabriela

Višniakov, Nikolaj

Visuri, Ville-Valtteri

Vitos, Levente

Viviani, Massimo

Vivo, Paola

Vizureanu, Petrică

Vlach, Martin

Vlaskin, Mikhail

Vlcak, Petr

Vokoun, David

Volkova, Olena

Vollertsen, Frank

Vora, Hitesh

Vorozhtsov, Alexander

Vrána, Radek

Vranceanu, Diana M.

Vrancken, Bey

Vrestal, Jan

Vukelic, Goran

Vuorinen, Esa

Wachowski, Marcin

Wachowski, Sebastian

Wagh, Priyesh

Wagner, Scott

Wagner, Stefan

Wajima, Takaaki

Wallbrink, Chris

Walvekar, Aditya A.

Wang, Jiangting

Wang, Xiaoqing

Wang, Yongxiang

Wanhill, Russell

Warmuzek, Małgorzata

Waryoba, Daudi R.

Waugh, David Garreth

Weaver, Jordan

Weber, Franz

Weber, Sebastian

Węglowski, Marek

Węgrzyn, Tomasz

Wei, Dongbin

Weidner, Anja

Weinberg, Annelie-Martina 
Weise, Joerg

Weise, Jörg

Weisheit, Andreas

Weiß, Sabine

Weißensteiner, Irmgard

Welle, Alexander

Westermann, Ida

Wettlaufer, Marc

Wexler, David

Wheeler, Robert

White, Jesse F.

Whittaker, Mark T.

Wieleba, Wojciech

Wierzba, Bartek

Wilcox, Paul

Wilding, Martin

Williams, John

Winczek, Jerzy

Winiczenko, Radosław

Wint, Natalie

Winther, Grethe

Wiora, Józef

Wiskel, J. Barry

Wisner, Brian

Wisniewski, Adam

Wlodarczyk, Renata

Woizeschke, Peer

Wojciechowski, Łukasz

Wojciechowski, Szymon

Wołczyński, Waldemar

Worrall, Stephen

Wozniak, Jarosław

Woźniak, Jarosław

Wróbel, Jan

Wróbel, Joanna

Wróbel, Piotr

Wróbel, Tomasz

$\mathrm{Wu}$, Hui

$\mathrm{Wu}$, Xijia

Wyszyński, Dominik

Xavier Gil, Javier

Xiao, Zhongmin

Xie, Haibo

Yagami, Kimitoshi

Yakymovych, Andriy

Yamabe, Junichiro

Yamamoto, Hajime

Yamanaka, Kenta

Yamasaki, Shigeto

Yan, Dayun

Yanaseko, Tetsuro

Yanushkevich, Zhanna
Yasnikov, Igor S.

Yates, Matthew

Yeo, In-Sung

Yeong, Wai Yee

$\mathrm{Yi}$, Sangbong

Yildirim, Halid

Yilmaz, Erol

Yin, Fei

Yo, Tomota

YONG-GI, Roh

Yoo, Dong Jin

Yoon, Seog-Young

Yoon, Yong Soo

Yoshida, Sanichiro

You, Tae-Soo

Yurchenko, Nikita

Yusenko, Kirill

Yusenko, Kirill V.

Zagórski, Ireneusz

Zaitsev, Alexandr A.

Zajec, Bojan

Zakowski, Krzysztof

Zamanzade, Mohammad

Zanella, Caterina

Zanotto, Federica

Zapryagaev, V. I.

Zdravecká, Eva

Zdunek, Joanna

Zeidler, Henning

Zelený, Martin

Zervaki, Anna D.

Zgodavová, Kristína

Zhang, Junxian

Zhang, Laichang

Zhang, Lai-Chang

Zhang, Sung-Uk

Zhang, Wencai

Zhang, Xiaoyuan

Zhang, Xinchang

Zhang, Zhe

Zhao, Xin

Zherebtsov, Sergey

Zhilyaev, Alexander

Zhong, Xiangyu

Zhou, J.

Zhou, Xiaowang

Zieliński, Adam

Zieliński, Andrzej

Zienert, Tilo

Ziewiec, Krzysztof

Zinoviev, Aleksandr

Zkria, Abdelrahman 
Żmudzki, Jarosław

Zolper, Thomas

Zoltán, Pászti

Zotti, Linda

Zubiaur, Pablo Pérez
Zucchi, Fabrizio

Zukowski, Witold

Žunda, Audrius

Zupanič, Franc

Zykova, Anna

(c) 2020 by the author. Licensee MDPI, Basel, Switzerland. This article is an open access article distributed under the terms and conditions of the Creative Commons Attribution (CC BY) license (http://creativecommons.org/licenses/by/4.0/). 